\title{
BMJ Open Cost-effectiveness of prehabilitation prior to elective surgery compared to usual preoperative care: protocol for a systematic review of economic evaluations
}

Tanja Rombey, Helene Eckhardt, Wilm Quentin

To cite: Rombey T, Eckhardt $\mathrm{H}$, Quentin W. Cost-effectiveness of prehabilitation prior to elective surgery compared to usual preoperative care: protocol for a systematic review of economic evaluations. BMJ Open 2020;10:e040262. doi:10.1136/ bmjopen-2020-040262

- Prepublication history and additional for this paper is available online. To view these files, please visit the journal online (http://dx.doi.org/10. 1136/bmjopen-2020-040262).

Received 11 May 2020 Revised 19 November 2020 Accepted 16 December 2020

Check for updates

(C) Author(s) (or their employer(s)) 2020. Re-use permitted under CC BY-NC. No commercial re-use. See rights and permissions. Published by BMJ.

Department of Health Care Management, Technische Universität Berlin, Berlin, Berlin, Germany

Correspondence to

Ms Tanja Rombey;

tanja.rombey@tu-berlin.de

\section{ABSTRACT}

Introduction Preoperative functional capacity is an important predictor of postoperative outcomes. Prehabilitation aims to optimise patients' functional capacity before surgery to improve postoperative outcomes. As prolonged hospital stay and postoperative complications present an avoidable use of healthcare resources, prehabilitation might also save costs. The aim of this systematic review is to investigate the cost-effectiveness of prehabilitation programmes for patients awaiting elective surgery compared with usual preoperative care. The results will be useful to inform decisions about the implementation of prehabilitation programmes and the design of future economic evaluations of prehabilitation programmes.

Methods and analysis We will search PubMed, Embase, the Centre for Reviews and Dissemination Database, the WHO International Clinical Trials Registry Platform and ClinicalTrials.gov for full or partial economic evaluations of preoperative prehabilitation programmes conducted in any population compared with usual preoperative care. Studies will be included regardless of the type, design and perspective of the economic evaluation, and their publication year, language or status. Initial searches were performed between 30 April and 4 May 2020.

Study selection, data extraction and assessment of the included studies' risk of bias and methodological quality will initially be performed by two independent reviewers and, if agreement was sufficiently high, by one reviewer. We will extract data regarding the included studies' basic characteristics, economic evaluation methods and costeffectiveness results.

A narrative synthesis will be performed. The primary endpoint will be cost-effectiveness based on cost-utility analyses. We will discuss heterogeneity between the studies and assess the risk of publication bias. The certainty of the evidence will be determined using the Grading of Recommendations, Assessment, Development and Evaluation approach.

Ethics and dissemination Ethics approval is not required as the systematic review will not involve human participants. We plan to present our findings at scientific conferences, pass them on to relevant stakeholder organisations and publish them in a peer-reviewed journal. PROSPERO registration number CRD42020182813
Strengths and limitations of this study

- A strength of this study is that the search strategy was developed involving an experienced information specialist using the Peer Review of Electronic Search Strategies 2015 Evidence-Based Checklist.

- Furthermore, the selection of electronic databases was based on research of the most efficient combination of healthcare databases that should be used to identify studies for systematic reviews of economic evaluations.

- The findings of this systematic review might be limited by the fact that economic evaluations are generally prone to publication bias, as they will usually only be performed if the intervention is effective.

- Furthermore, the quality of the evidence generated through this systematic review will depend on the risk of bias/methodological quality and reporting quality of the included studies.

- Lastly, as both prehabilitation programmes and economic evaluations are by nature heterogeneous, it might not be possible to draw firm conclusions from this systematic review, which are transferable to a range of health systems.

\section{BACKGROUND}

\section{Rationale}

Each year, millions of surgical procedures are performed worldwide. For example, in Germany, 17 million surgeries requiring hospitalisation were performed in $2018 .{ }^{1}$ A major concern in the surgical context is the prevention of postoperative complications, which are devastating for patients and burdensome for health systems as they present avoidable use of healthcare resources, ${ }^{2}$ for example, through a prolonged length of hospital stay or readmissions. Preoperative functional capacity is an important predictor of postoperative outcomes, ${ }^{3-6}$ therefore, it can be hypothesised that prehabilitation 


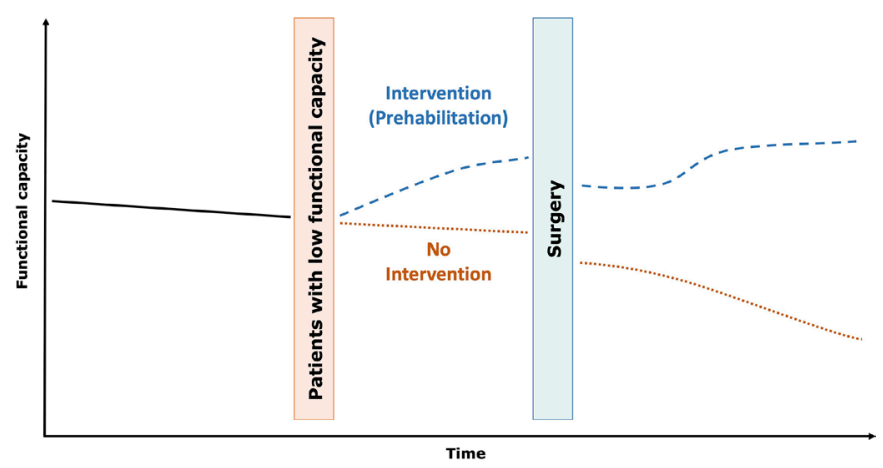

Figure 1 Events pathway diagram of patients with low functional capacity. Own figure based on Birkelbach et $a l^{52}$.

prior to elective surgery might prevent complications and thereby save costs.

While rehabilitation has long been an essential part of healthcare in developed countries, ${ }^{7}$ prehabilitation has only received increased attention in the past two decades. ${ }^{8}$ The word consists of the prefix pre and the noun rehabilitation and is defined as the process of 'enhancing an individual's functional capacity to enable him or her to withstand a forthcoming stressor'. ${ }^{9}$ The idea behind prehabilitation is to begin the rehabilitation process to optimise an individual's functional capacity before the stressor, for example, a surgery, takes place and thus to enhance outcomes and recovery afterwards, ${ }^{10}$ see figure 1. Prehabilitation programmes may include one or more modalities, such as medical optimisation (eg, smoking cessation or control of blood glucose), physical exercise and promotion of physical activity, nutritional support or psychological support and are usually provided by a multidisciplinary team. ${ }^{9}$

The first randomised controlled trial (RCT) on prehabilitation prior to elective surgery was published in 2000. ${ }^{11}$ It investigated the effect of a multimodal preoperative intervention for low-risk patients awaiting elective coronary artery bypass graft surgery compared with usual care. The authors found that prehabilitation reduced length of hospital stay by 1 day, resulting in an approximate net saving of \$C133 per patient per day. ${ }^{11}$ Their RCT was followed by a large number of primary studies whose results have been synthesised in various systematic reviews, the majority of which looked at abdominal surgery. ${ }^{12-22}$

The cost-effectiveness of an intervention, that is, its value for money, is an important factor for health policymakers deciding about the implementation of a new programme. ${ }^{23}$ However, despite the growing interest in prehabilitation programmes by healthcare professionals and policy-makers, only a subset of studies has evaluated the cost-effectiveness of prehabilitation. To date, there is no systematic review that provides an overview of the cost-effectiveness of prehabilitation programmes across different surgical disciplines. Hence, it still needs to be determined if prehabilitation prior to elective surgery is cost-effective. ${ }^{9}$
This systematic review is part of a larger project that investigates prehabilitation of frail or prefrail patients before elective surgery as a new model of care in Germany. ${ }^{24}$ An RCT enrolling more than 1400 patients and an accompanying economic evaluation is planned. Therefore, we are not only interested in the findings of previous economic evaluations of prehabilitation programmes prior to elective surgery but also in their methods to guide our own economic evaluation.

\section{Objective}

The aim of this systematic review is to answer the question: What is the cost-effectiveness of prehabilitation programmes for patients awaiting elective surgery compared with usual preoperative care?

Our objectives are to identify all eligible economic evaluations, assess their validity and systematically present their characteristics, methods and findings to inform decisions about the implementation of prehabilitation programmes and guide the design of future rigorous economic evaluations of prehabilitation programmes.

\section{METHODS}

The design of our systematic review followed the five-step approach to prepare a systematic review of economic evaluations for informing evidence-based healthcare decisions. ${ }^{25}$ Furthermore, we consulted the Cochrane Handbook for Systematic Reviews of Interventions ${ }^{26}$ and guidance for undertaking reviews in healthcare by the Centre for Reviews and Dissemination (CRD) of the University of York, in particular chapter $5 .{ }^{27}$ This protocol is reported in accordance with the Preferred Reporting Items for Systematic Review and Meta-Analysis Protocols (PRISMA-P) 2015 statement $^{2829}$ (see online supplemental appendix 1).

\section{Registration}

After checking that there is no similar published or ongoing systematic review, we have submitted a record for our systematic review on PROSPERO on 30 April 2020 (CRD42020182813).

\section{Eligibility criteria}

The study inclusion and exclusion criteria can be found in table 1.

As we aim to provide a comprehensive overview of the available evidence on the cost-effectiveness of prehabilitation, we decided to include both full and partial economic evaluations as well as economic evaluations based on both RCTs and non-randomised studies of interventions (NRSI). While full economic evaluations should generally be preferred over partial economic evaluations, ${ }^{30}$ partial economic evaluations might still be useful for decisionmakers to understand the costs and consequences of an intervention. ${ }^{31}$

\section{Information sources}

We will search the following electronic databases and trial registries from their inception: 


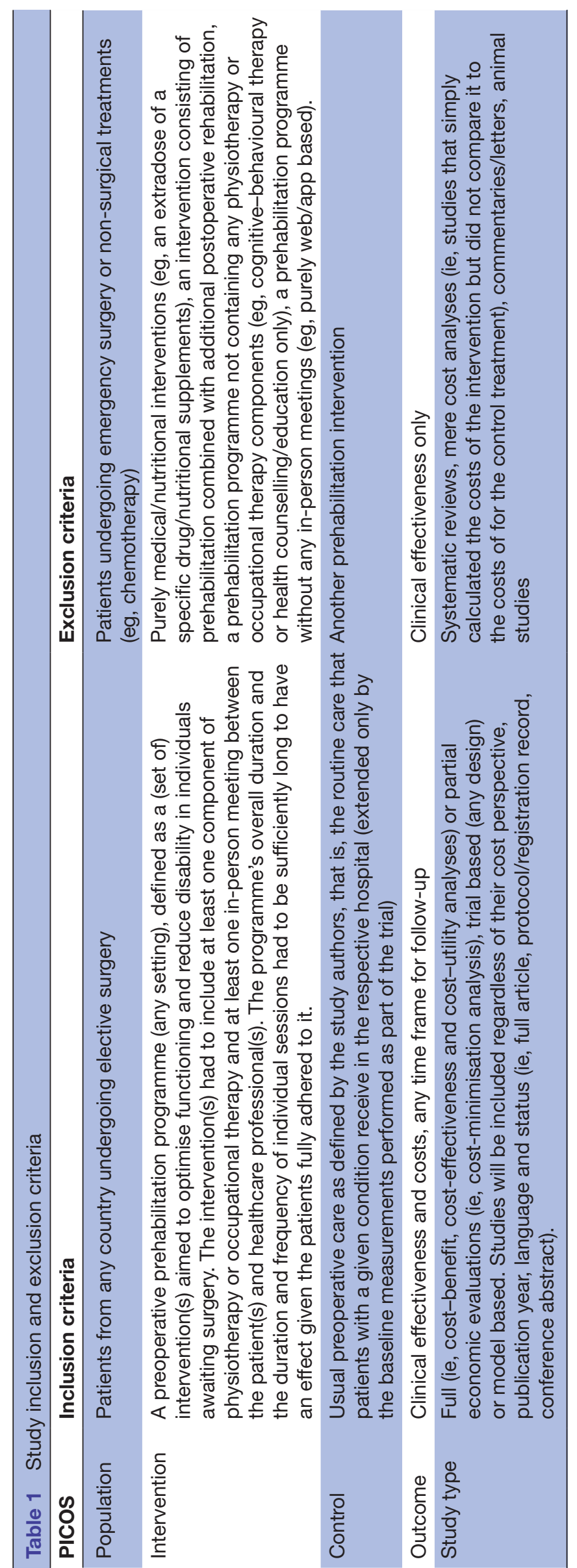

- PubMed (via PubMed), initial search date 30 April 2020.

- Embase (via Embase), initial search date 30 April 2020.

- CRD Database (containing the Health Technology Assessment (HTA) database and archives of the Database of Abstracts of Reviews of Effects and the National Health Service Economic Evaluation Database) (via CRD Database), initial search date 4 May 2020.

- WHO International Clinical Trials Registry Platform(ICTRP), not searched yet as the ICTRP Search Portal was temporarily not accessible from outside WHO due to the heavy traffic generated by the COVID-19 outbreak ${ }^{32}$

- ClinicalTrials.gov (although ClinicalTrials.gov is included in the WHO ICTRP, it is recommended that it is searched separately due to additional features ${ }^{26}$ ), initial search date 4 May 2020.

The selection of electronic databases was based on recommendations by the CRD and a study by Arber et al who found that the most efficient combination of healthcare databases was Embase, the HTA database and PubMed. ${ }^{33}$

In addition, we will screen the reference lists of any relevant systematic reviews on prehabilitation identified and of the included studies, and perform a forward citation search in Web of Science (WoS), or in Google Scholar if a study was not indexed in WoS. To identify ongoing or (yet) unpublished studies (grey literature), we will search Open Access Theses and Dissertations and the DARTEurope E-theses Portal. We will also contact the authors of the included studies who we consider content experts about whether they know of any further eligible studies.

\section{Search strategy}

Following initial scoping searches, the draft search strategies for PubMed and Embase were developed by one reviewer (TR). An experienced information specialist (HE) reviewed the draft search strategies using the Peer Review of Electronic Search Strategies 2015 EvidenceBased Checklist.

The revised search strategy (see online supplemental appendix 2) consists of three sets of terms, relating to the population, intervention and study type, respectively: (1) terms to search for the population (such as preoperative), (2) terms to search for the intervention (such as exercise) and (3) terms to search for the economic evaluations (such as cost). The term prehabilitation is included in the set of terms relating to the population as well as the intervention. Both, controlled vocabulary (such as Medical Subject Headings (MeSH) terms in MEDLINE) and freetext terms (including truncations where appropriate) are used and no limits will be applied. We will report the final search strategies following the PRISMA Search Reporting Extension (PRISMA-S).$^{34}$

To identify economic evaluations in MEDLINE, we will use the following terms: cost[tiab] OR costs[tiab] economic* [tiab] OR budget*[tiab] OR "Costs and cost analysis"[MeSH] 
OR "Exercise/economics"[Mesh] OR "Rehabilitation/economics"[Mesh]. These terms were inspired by a filter by Wilczynski $e t a l^{35}$ that had the best optimisation of sensitivity and specificity in identifying economic evaluations in MEDLINE according to a study by Glanville et al. ${ }^{36}$ To identify economic evaluations in Embase, we will use a filter (adapted for the Embase search surface) by McKinlay et $a l^{37}$ that had the best optimisation of sensitivity and specificity in identifying economic evaluations in Embase according to Glanville et $a \hat{l}^{6}$ : cost:ti,ab, $k w O R$ costs:ti,ab,kw. Analogue to the PubMed search strategy, these terms were supplemented by the following terms: economic*:ti,ab, kw OR budget ${ }^{*}: t i, a b, k w$ OR 'economic aspect'। exp.

Once the first round of study selection has been completed, we will calculate the search strategies' relative recall (identified eligible records/all eligible records indexed in the database) for the electronic databases. If one of the search strategies' relative recall will be below $90 \%$, we will revise it and perform a new search in that database. Weekly alerts for new studies identified with our search strategy will be activated for PubMed (using MyNCBI) and Embase. All searches will be repeated at completion of the systematic review to ensure that it is up to date and any new studies included in a second round of study selection can be incorporated before publication.

\section{Data management}

All records will be imported to EndNote X9.3.2 (Clarivate Analytics, Philadelphia, Pennsylvania USA) where they will be deduplicated and screened for eligibility. Full-text articles will also be stored in EndNote.

\section{Selection process}

Two reviewers (TR and HE) will screen a random $10 \%$ sample of all unique records based on their titles and abstracts and discuss their results until consensus has been reached. If agreement between them was sufficiently high (at least $80 \%$ raw agreement), the remaining records will be screened by one reviewer (TR). If agreement was below $80 \%$, another $10 \%$ sample will be screened by the same two reviewers and the process will be repeated. We will mark any relevant systematic reviews on prehabilitation.

We will retrieve the full-text articles for all records deemed potentially eligible after title/abstract screening and of relevant systematic reviews on prehabilitation. Two reviewers (TR and HE) will independently screen all fulltext articles of potentially eligible studies and capture reasons for exclusion. Eligible articles will then be mapped to studies (as the unit of interest). Records of ongoing studies (eg, protocol publications or registration records) will be included, as we are interested in the methods of ongoing studies, too. The references of all eligible studies and all relevant systematic reviews on prehabilitation will be screened for further eligible studies.

Results of the study selection process will be displayed in form of a flow diagram. ${ }^{28} \mathrm{~A}$ list of articles excluded after full-text screening with reasons for exclusion will be provided.

\section{Data collection process}

All eligible studies will be checked for errata or retractions before data extraction. Data will be extracted into a standardised Excel sheet. One reviewer (TR) will pilot the extraction sheet using $n=2$ randomly selected studies to test its user-friendliness and completeness, then two reviewers (TR, HE/WQ) will perform a calibration exercise by independently extracting the data of a random $20 \%$-sample of the included studies. If less than $\mathrm{n}=15$ studies are included, $\mathrm{n}=3$ studies will randomly be selected for independent data extraction by two reviewers. Discrepancies will be discussed until consensus has been reached and the extraction sheet will be revised where necessary. If agreement between the reviewers was sufficiently high (at least $80 \%$ raw agreement), the data of the remaining studies will be extracted by one reviewer (TR). If agreement was below $80 \%$, the process will be repeated.

We will contact the study authors via email to obtain missing data or resolve any uncertainties regarding their data. A reminder email will be sent after 2 weeks if necessary. Missing data will not be imputed if emails remain unanswered. Unresolved uncertain data will be marked as such.

\section{Data items}

We will extract data on various items (see table 2).

In addition, we will extract details on the methods of the economic evaluations (see table 3 ).

This selection of items was informed by Wijnen et al. ${ }^{30} \mathrm{~A}$ draft data extraction form can be found in online supplemental appendix 3 .

\section{Outcomes and prioritisation}

Our primary outcome is the cost-effectiveness of prehabilitation prior to elective surgery based on results from costutility analyses, as these provide a cost-effectiveness measure (cost per quality-adjusted life year (QALY) gained) that is comparable across disciplines and for which willingness to pay thresholds are available for several countries. Therefore, they are most meaningful to health policy-makers.

Secondary outcomes are the clinical effectiveness- and cost outcomes of prehabilitation prior to elective surgery based on results from other types of economic evaluations. We will only consider clinical effectiveness outcomes that are patient-relevant (eg, mortality, morbidity or quality of life). Surrogates (eg, duration of surgery or laboratory parameters) will not be considered. If there are different cost outcomes (eg, costs during hospital stay, costs following hospital stay and total costs), we will only consider the total costs. If no total costs are reported, we will aim to calculate them based on the cost data provided.

\section{Risk of bias in and methodological quality of individual} studies

Trial-based economic evaluations: We will assess the risk of bias on study level using the current gold standard 
Table 2 Data extraction items

\begin{tabular}{|c|c|}
\hline Item & Specification \\
\hline Study ID & $\begin{array}{l}\text { Study acronym/first author's last } \\
\text { name, publication year }\end{array}$ \\
\hline Registration & Registration no (eg, NCT no) \\
\hline Source of funding & $\begin{array}{l}\text { Non-profit, for-profit, mixed, unclear, } \\
\text { no funding; not stated } \\
\text { If funded: extract name of funder }\end{array}$ \\
\hline
\end{tabular}

Competing interests No, yes; not stated If yes: specify

$\begin{array}{ll}\text { Publication type } & \begin{array}{l}\text { Full article/HTA report, protocol/ } \\ \text { registration record, conference } \\ \text { abstract }\end{array} \\ \text { Location } & \begin{array}{l}\text { Country and city where the } \\ \text { investigation was performed } \\ \text { If multicentre: Extract all cities and } \\ \text { countries }\end{array}\end{array}$

$\begin{array}{ll}\text { Enrolment period } & \mathrm{mm} / \mathrm{yyyy} \text { to } \mathrm{mm} / \mathrm{yyyy} \\ \text { Length of follow-up } & \text { In months }\end{array}$

\begin{tabular}{|c|c|}
\hline Population & $\begin{array}{l}\text { Description of study participants' } \\
\text { underlying disease and type of surgery } \\
\text { Inclusion and exclusion criteria } \\
\text { If study has been completed: } \\
\text { No of randomised patients (in total, } \\
\text { per group) } \\
\text { Age, gender, comorbidities of patients }\end{array}$ \\
\hline Intervention & $\begin{array}{l}\text { Description of the study intervention } \\
\text { (setting, modality, overall duration, } \\
\text { frequency, session duration, healthcare } \\
\text { staff performing the intervention) }\end{array}$ \\
\hline Control & $\begin{array}{l}\text { Description of the control intervention } \\
\text { (eg, description of the usual } \\
\text { preoperative care if reported) }\end{array}$ \\
\hline Outcomes & $\begin{array}{l}\text { Description of the outcomes (including } \\
\text { effects, costs and cost-effectiveness) } \\
\text { If study has been completed: } \\
\text { Results on effects in disaggregated } \\
\text { and aggregated form (with Cls) } \\
\text { Results on costs in disaggregated } \\
\text { and aggregated form (with Cls) } \\
\text { Results on cost-effectiveness (eg, } \\
\text { incremental cost-effectiveness } \\
\text { ratios) } \\
\text { Study authors' conclusion } \\
\text { regarding the intervention's cost- } \\
\text { effectiveness (copied verbatim) }\end{array}$ \\
\hline
\end{tabular}

HTA, Health Technology Assessment; ID, Identifier; NCT,

ClinicalTrials.gov identifier.

tool, which currently would be the Cochrane RoB V.2.0 tool $^{38}$ for RCTs and the ROBINS-I tool ${ }^{39}$ for NRSI. In addition, we will assess the methodological quality of all full economic evaluations using the Consensus on Health Economic Criteria checklist. ${ }^{40}$ For model-based studies, we will only assess the methodological quality of the economic evaluation using the International Society for Pharmacoeconomics and Outcomes Research checklist. ${ }^{41}$
Two reviewers (TR, HE/WQ) will independently assess a random $20 \%$-sample of the included studies. If less than $n=15$ studies are included, $n=3$ studies will randomly be selected for assessment. If agreement between them was sufficiently high (at least $80 \%$ raw agreement), the remaining studies will be assessed by one reviewer (TR). If agreement was below $80 \%$, another $20 \%$ sample will be assessed by the same two reviewers and the process will be repeated.

\section{Data synthesis}

We will perform a meta-analysis using standard methods ${ }^{26}$ if we identify two or more methodologically homogenous studies reporting on the same outcome. However, as both prehabilitation programmes ${ }^{9}$ and economic evaluations ${ }^{27}$ are by nature rather heterogeneous, we do not expect to be able to meta-analyse the included studies' results. Thus, we will perform a narrative synthesis by comparing their results in detail in table format and summarising them in text form. We will present the included studies' basic characteristics (including details on the prehabilitation programmes) and economic evaluation methods. For completed studies, we will also present the results of our risk of bias/ methodological quality assessment and the studies' cost-effectiveness results (presented alongside their overall risk of bias/methodological quality).

Results will be presented in aggregated (eg, as an incremental cost-effectiveness ratio) and disaggregated form in natural units if possible. For example, the primary outcome (cost-effectiveness of prehabilitation based on a cost-utility analysis) will be reported as cost per QALY gained and as change in health-related quality of life, length of life, quantities of resource use and unit costs. This is to allow decision-makers to apply the results to their own context and to facilitate the reuse of these data as inputs to of future model-based economic evaluations. ${ }^{42}$ Costs will be reported in the original monetary units as well as converted to 2020 Euros. For conversion, we will use the Campbell and Cochrane Economics Methods Group and the Evidence for Policy and Practice Information and Coordinating Centre Cost Converter, ${ }^{43}$ a free web-based tool for adjusting estimates of cost expressed in one currency and price year to a specific target currency and price year. We will not aim to rank the studies based on their cost-effectiveness results, but we may present their results in cost-effectiveness planes where common metrics were used.

Studies will be ordered alphabetically by study identifier (ID). Depending on the number of included studies, we may group studies according to their setting, population, intervention or methods. We will narratively discuss heterogeneity between the studies.

\section{Metabias(es)}

We will address selective reporting within studies by comparing study reports with their study protocols whenever such are available. 


\begin{tabular}{|c|c|}
\hline Item & Specification \\
\hline Type of analysis & $\begin{array}{l}\text { Cost-minimisation analysis, cost-consequence analysis, cost-benefit analysis, cost- } \\
\text { effectiveness analysis, cost-utility analysis; other (with description) }\end{array}$ \\
\hline Design of economic evaluation & $\begin{array}{l}\text { Trial based, model based } \\
\text { If trial based: RCT or NRSI } \\
\text { If model based: Markov, decision tree and discrete event simulation }\end{array}$ \\
\hline Cost perspective & $\begin{array}{l}\text { Societal perspective, healthcare payer perspective, healthcare provider perspective, patient } \\
\text { perspective; other (with description)) }\end{array}$ \\
\hline Time horizon & For effects and costs; in months \\
\hline Effects & $\begin{array}{l}\text { Data source of effects } \\
\text { Measurement of effects } \\
\text { Valuation of effects }\end{array}$ \\
\hline Costs & $\begin{array}{l}\text { Type (direct/indirect) } \\
\text { Approach (top-down/bottom-up) } \\
\text { Data source of resource use } \\
\text { Measurement of resource use } \\
\text { Valuation (methods used to calculate unit costs) }\end{array}$ \\
\hline Missing data & Handling of missing data \\
\hline Discounting & No, yes (with description of discount rate for effects and costs) \\
\hline Inflation rate & No, yes (with description) \\
\hline Reference year and currency & State year and currency \\
\hline Statistical analysis & $\begin{array}{l}\text { Details of the analysis of cost-effectiveness } \\
\text { For model-based studies: model assumptions }\end{array}$ \\
\hline Uncertainty & $\begin{array}{l}\text { Details of the analyses of uncertainty (eg, statistical comparison, bootstrapping, sensitivity } \\
\text { analysis(one way, multiway), threshold analysis (eg, using a cost-effectiveness acceptability } \\
\text { curve), analysis of extremes and best/worst case analysis) and probabilistic sensitivity } \\
\text { analysis }\end{array}$ \\
\hline Willingness-to-pay threshold & Sum per unit of health outcome (eg, 20000 pound sterling per QALY) \\
\hline
\end{tabular}

NRSI, non-randomised studies of interventions; QALY, Quality-adjusted life year; RCT, randomised controlled trial.

To address publication bias across studies, we will search comprehensively for relevant trial registration records and study protocols. We will contact authors if their record/protocol implies that the study has already been completed to follow up on the study's status.

\section{Confidence in cumulative evidence and transferability}

We will determine the quality (high, moderate, low or very low) of the evidence for each cost-effectiveness outcome following the Grading of Recommendations, Assessment, Development and Evaluation (GRADE) approach, ${ }^{44}$ particularly following part 10 of the GRADE Guidelines (Considering Resource Use and Rating the Quality of Economic Evidence) ${ }^{45}$ We will summarise our assessment in form of a GRADE evidence profile and in Summary of Findings tables using disaggregated data. Furthermore, we will discuss the transferability of our results to different health systems using a checklist by Welte $e t$ al. ${ }^{46}$

\section{Study dates}

The systematic review is currently ongoing. We started with the conception and preliminary searches on 1 April 2020. The anticipated date of completion is 31 March 2021.
We plan to update this systematic review in summer 2022, as we expect that our own economic evaluation and some of the ongoing studies that we might identify in this initial systematic review will have been completed by then.

\section{Patient and public involvement}

Patients or the public were not involved in the design, or conduct, or reporting, or dissemination plans of our research.

\section{Ethics and dissemination}

Our systematic review will not involve human participants or contain personal and/or medical information about an identifiable living individual. Therefore, ethics approval or consent to participate is not required.

We plan to present our findings at scientific conferences, pass them on to relevant stakeholder organisations and publish them in a peer-reviewed journal. We will report our systematic review in accordance with the Preferred Reporting Items for Systematic reviews and Meta-Analyses (PRISMA) ${ }^{47}$ or the updated PRISMA Statement ${ }^{49}$ if available by then. Furthermore, we will disseminate links to this protocol, the PROSPERO record 
and the final publication via social media (Twitter and ResearchGate).

\section{Amendments}

Important protocol amendments will be documented in the systematic review's PROSPERO record. A (dated) new version of the record with short explanation of the amendment will then be published.

\section{DISCUSSION}

This systematic review of economic evaluations will provide its readers with a summary and synthesis of studies that have evaluated or will evaluate the cost-effectiveness of prehabilitation prior to elective surgery compared with usual preoperative care. Our systematic review will be helpful both for decision-makers who consider the implementation of a prehabilitation programme as well as for researchers aiming to perform an economic evaluation of a prehabilitation programme in the future ${ }^{50}$ As we will include studies on patients from all surgical disciplines without restrictions, we may be able to identify knowledge gaps for certain groups of patients, for whom no studies were found or who did not meet the studies' eligibility criteria.

We anticipate a number of limitations. First, economic evaluations are generally prone to publication bias, as many study authors will only consider performing an economic evaluation if there is conclusive evidence that the study intervention is more effective than the control intervention. ${ }^{42}$ We will address this issue by searching comprehensively for relevant trial registration records and study protocols and following-up on any studies that are supposed to be completed but have not yet been published.

Second, as there is currently no gold-standard tool to assess the methodological quality of economic evaluations,${ }^{30}$ we had to choose the aforementioned tools based on their user friendliness and feasibility in the context of our systematic review. Furthermore, instead of having to use two tools for trial-based and model-based economic evaluations, a single validated tool to assess the risk of bias in both types of economic evaluations would be much welcomed. In addition, both the Cochrane RoB V.2.0 tool $^{38}$ and the ROBINS-I tool ${ }^{39}$ are relatively new tools that still need to be validated. ${ }^{51}$

Third, the quality of the evidence generated through this systematic review will depend on the risk of bias/methodological quality of the included studies. For example, in case of prehabilitation, it is not usually possible to blind patients or investigators to the intervention. We will discuss the risk of bias/methodological quality of the included studies when reporting their results and make recommendations of how to improve the validity of economic evaluations' findings.

Fourth, trial-based studies that present partial economic evaluations and/or are reported alongside the trial results are likely to report their economic evaluation methods only briefly. Therefore, we might not be able to extract data in the same detail for them as for full economic evaluations/trial-based economic evaluations that were published separately. Lastly, as both prehabilitation programmes and economic evaluations are usually heterogeneous in their design, it might not be possible to draw firm conclusions from this systematic review, which are transferable to a range of health systems.

Contributors TR and WQ conceptualised the review. TR drafted the protocol. HE and WQ revised the protocol. All authors were involved in the conception of the protocol, approved the final version of the manuscript to be published and agree to be accountable for all aspects of the planned systematic review.

Funding The systematic review is part of a larger project which is supported by the Innovation Fund coordinated by the Innovation Committee of the Federal Joint Committee in Germany (Innovationsausschuss beim Gemeinsamen Bundesausschuss (G-BA)), grant number 01NVF18024. Furthermore, we acknowledge support by the Open Access Publication Fund of TU Berlin who covered the Article Processing Charge for this protocol.

Disclaimer The funders had no role in developing the protocol.

Competing interests None declared.

Patient consent for publication Not required.

Provenance and peer review Not commissioned; externally peer reviewed.

Supplemental material This content has been supplied by the author(s). It has not been vetted by BMJ Publishing Group Limited (BMJ) and may not have been peer-reviewed. Any opinions or recommendations discussed are solely those of the author(s) and are not endorsed by BMJ. BMJ disclaims all liability and responsibility arising from any reliance placed on the content. Where the content includes any translated material, BMJ does not warrant the accuracy and reliability of the translations (including but not limited to local regulations, clinical guidelines, terminology, drug names and drug dosages), and is not responsible for any error and/or omissions arising from translation and adaptation or otherwise.

Open access This is an open access article distributed in accordance with the Creative Commons Attribution Non Commercial (CC BY-NC 4.0) license, which permits others to distribute, remix, adapt, build upon this work non-commercially, and license their derivative works on different terms, provided the original work is properly cited, appropriate credit is given, any changes made indicated, and the use is non-commercial. See: http://creativecommons.org/licenses/by-nc/4.0/.

\section{REFERENCES}

1 Statistisches Bundesamt. Operationen und Prozeduren der vollstationären Patientinnen und Patienten in Krankenhäusern (4-Steller) - 2018. Wiesbaden: Statistisches Bundesamt (Destatis), 2019. https://www.destatis.de/DE/Themen/Gesellschaft-Umwelt/ Gesundheit/Krankenhaeuser/Publikationen/DownloadsKrankenhaeuser/operationen-prozeduren-5231401187014.html

2 Patel AS, Bergman A, Moore BW, et al. The economic burden of complications occurring in major surgical procedures: a systematic review. Appl Health Econ Health Policy 2013;11:577-92.

3 Moonesinghe SR, Mythen MG, Grocott MPW. Patient-Related risk factors for postoperative adverse events. Curr Opin Crit Care 2009;15:320-7

4 Snowden CP, Prentis J, Jacques B, et al. Cardiorespiratory fitness predicts mortality and hospital length of stay after major elective surgery in older people. Ann Surg 2013;257:999-1004.

5 Prentis JM, Trenell MI, Vasdev N, et al. Impaired cardiopulmonary reserve in an elderly population is related to postoperative morbidity and length of hospital stay after radical cystectomy. BJU Int 2013;112:E13-19.

6 Sinclair RCF, Phillips AW, Navidi M, et al. Pre-Operative variables including fitness associated with complications after oesophagectomy. Anaesthesia 2017;72:1501-7.

7 World Health Organization. Rehabilitation in health systems. Geneva: World Health Organization. Licence: CC BY-NC-SA 3.0 IGO, 2017.

8 Carli F, Zavorsky GS. Optimizing functional exercise capacity in the elderly surgical population. Curr Opin Clin Nutr Metab Care 2005;8:23-32.

9 Banugo P, Amoako D. Prehabilitation. BJA Educ 2017;17:401-5. 
10 Wynter-Blyth V, Moorthy K. Prehabilitation: preparing patients for surgery. BMJ 2017;358:j3702.

11 Arthur HM, Daniels C, McKelvie R, et al. Effect of a preoperative intervention on preoperative and postoperative outcomes in low-risk patients awaiting elective coronary artery bypass graft surgery. A randomized, controlled trial. Ann Intern Med 2000;133:253-62.

12 Gillis C, Buhler K, Bresee L, et al. Effects of Nutritional Prehabilitation, With and Without Exercise, on Outcomes of Patients Who Undergo Colorectal Surgery: A Systematic Review and Metaanalysis. Gastroenterology 2018;155:391-410.

13 Moran J, Guinan E, McCormick P, et al. The ability of prehabilitation to influence postoperative outcome after intra-abdominal operation: a systematic review and meta-analysis. Surgery 2016;160:1189-201.

14 Hijazi Y, Gondal U, Aziz O. A systematic review of prehabilitation programs in abdominal cancer surgery. Int J Surg 2017;39:156-62.

15 Hughes MJ, Hackney RJ, Lamb PJ, et al. Prehabilitation before major abdominal surgery: a systematic review and meta-analysis. World $\mathrm{J}$ Surg 2019;43:1661-8.

16 Luther A, Gabriel J, Watson RP, et al. The impact of total body prehabilitation on post-operative outcomes after major abdominal surgery: a systematic review. World J Surg 2018;42:2781-91.

17 Bolshinsky V, Li MH-G, Ismail H, et al. Multimodal prehabilitation programs as a bundle of care in gastrointestinal cancer surgery: a systematic review. Dis Colon Rectum 2018;61:124-38.

18 Looijaard SMLM, Slee-Valentijn MS, Otten RHJ, et al. Physical and nutritional prehabilitation in older patients with colorectal carcinoma: a systematic review. J Geriatr Phys Ther 2018;41:236-44.

19 Bruns ERJ, van den Heuvel B, Buskens CJ, et al. The effects of physical prehabilitation in elderly patients undergoing colorectal surgery: a systematic review. Colorectal Dis 2016;18:O267-77.

20 Thomas G, Tahir MR, Bongers BC, et al. Prehabilitation before major intra-abdominal cancer surgery: a systematic review of randomised controlled trials. Eur J Anaesthesiol 2019;36:933-45.

21 Heger P, Probst P, Wiskemann J. A systematic review and metaanalysis of physical exercise prehabilitation in major abdominal surgery (prospero 2017 CRD42017080366). J Gastrointest Surg 2019.

22 Teo JYK, Turner R, Self M. Effect of exercise prehabilitation on functional status of patients undergoing bowel resection: a systematic review. ANZ J Surg 2020;90:693-701.

23 Lavis J, Davies H, Oxman A, et al. Towards systematic reviews that inform health care management and policy-making. J Health Serv Res Policy 2005;10 Suppl 1:35-48.

24 Innovationsausschuss. PRÄP-GO - Prähabilitation von älteren Patienten mit Gebrechlichkeitssyndrom vor elektiven Operationen. Berlin: Gemeinsamer Bundesausschuss, 2019. Available: https:// innovationsfonds.g-ba.de/projekte/neue-versorgungsformen/ praep-go-praehabilitation-von-aelteren-patienten-mit-gebrechl ichkeitssyndrom-vor-elektiven-operationen.276 [Accessed 14 April 2020].

25 van Mastrigt GAPG, Hiligsmann M, Arts JJC, et al. How to prepare a systematic review of economic evaluations for informing evidencebased healthcare decisions: a five-step approach (part 1/3). Expert Rev Pharmacoecon Outcomes Res 2016;16:689-704.

26 Higgins JPT TJ, Chandler J, Cumpston M. Cochrane Handbook for systematic reviews of interventions version 6.0 (updated July 2019). Cochrane, 2019. https://training.cochrane.org/cochrane-handbooksystematic-reviews-interventions

27 Centre for Reviews and Dissemination University of York. Systematic Reviews: CRD's guidance for undertaking reviews in health care. CRD, University of York: York, 2009.

28 Moher D, Shamseer L, Clarke M, et al. Preferred reporting items for systematic review and meta-analysis protocols (PRISMA-P) 2015 statement. Syst Rev 2015;4:1.

29 Shamseer L, Moher D, Clarke M, et al. Preferred reporting items for systematic review and meta-analysis protocols (PRISMA-P) 2015: elaboration and explanation. BMJ 2015;350:97647.

30 Wijnen B, Van Mastrigt G, Redekop WK, et al. How to prepare a systematic review of economic evaluations for informing evidencebased healthcare decisions: data extraction, risk of bias, and transferability (part 3/3). Expert Rev Pharmacoecon Outcomes Res 2016;16:723-32.
31 Drummond MF, Sculpher MJ, Claxton K. Methods for the economic evaluation of health care programmes. 4th. Oxford University Press: Oxford, 2015

32 World Health Organization. International clinical trials registry platform (ICTRP). Geneva: World Health Organization, 2020. https:// www.who.int/ictrp/search/en/

33 Arber M, Glanville J, Isojarvi J, et al. Which databases should be used to identify studies for systematic reviews of economic evaluations? Int J Technol Assess Health Care 2018;34:547-54

34 et alRethlefsen M, Ayala AP, Kirtley S. PRISMA-S: PRISMA search reporting extension 2019. Available: https://osf.io/ygn9w/ [Accessed 14 April 2020].

35 Wilczynski NL, Haynes RB, Lavis JN, et al. Optimal search strategies for detecting health services research studies in MEDLINE. CMAJ 2004:171:1179-85.

36 Glanville J, Kaunelis D, Mensinkai S. How well do search filters perform in identifying economic evaluations in MEDLINE and EMBASE. Int J Technol Assess Health Care 2009;25:522-9.

37 McKinlay RJ, Wilczynski NL, Haynes RB, et al. Optimal search strategies for detecting cost and economic studies in EMBASE. BMC Health Serv Res 2006;6:67.

38 Sterne JAC, Savović J, Page MJ, et al. RoB 2: a revised tool for assessing risk of bias in randomised trials. BMJ 2019:366:14898.

39 Sterne JA, Hernán MA, Reeves BC, et al. ROBINS-I: a tool for assessing risk of bias in non-randomised studies of interventions. BMJ 2016;355:i4919.

40 Evers S, Goossens M, de Vet $\mathrm{H}$, et al. Criteria list for assessment of methodological quality of economic evaluations: consensus on health economic criteria. Int J Technol Assess Health Care 2005;21:240-5.

41 Jaime Caro J, Eddy DM, Kan H, et al. Questionnaire to assess relevance and credibility of modeling studies for informing health care decision making: an ISPOR-AMCP-NPC good practice Task force report. Value Health 2014:17:174-82.

42 Shemilt IAP, Graybill E, Craig D. on behalf of the Campbell and Cochrane Economics Methods Group. Chapter 20: Economic evidence. In: Higgins JPT, Thomas J, Chandler J, eds. Cochrane Handbook for systematic reviews of interventions version 6.0 (updated July 2019). Cochrane, 2019. www.training.cochrane.org/ handbook

43 Shemilt I, Thomas J, Morciano M. A web-based tool for adjusting costs to a specific target currency and price year. Evidence \& Policy: A Journal of Research, Debate and Practice 2010;6:51-9.

44 et alSchünemann $\mathrm{H}$, Brożek J, Guyatt G. Handbook for grading the quality of evidence and the strength of recommendations using the grade approach, 2013. Available: https://gdt.gradepro.org/app/ handbook/handbook.html [Accessed 14 April 2020].

45 Brunetti M, Shemilt I, Pregno S, et al. Grade guidelines: 10. considering resource use and rating the quality of economic evidence. J Clin Epidemiol 2013;66:140-50.

46 Welte R, Feenstra T, Jager $\mathrm{H}$, et al. A decision chart for assessing and improving the transferability of economic evaluation results between countries. Pharmacoeconomics 2004;22:857-76.

47 Moher D, Liberati A, Tetzlaff J, et al. Preferred reporting items for systematic reviews and meta-analyses: the PRISMA statement. BMJ 2009;339:b2535.

48 Liberati A, Altman DG, Tetzlaff J, et al. The PRISMA statement for reporting systematic reviews and meta-analyses of studies that evaluate healthcare interventions: explanation and elaboration. $B M J$ 2009;339:b2700.

49 Page MJ. Updating the PRISMA reporting guideline for systematic reviews and meta-analyses 2018. Available: https://osf.io/p93ge/ [Accessed 14 April 2020].

50 Luhnen M, Prediger B, Neugebauer EAM, et al. Systematic reviews of economic evaluations in health technology assessment: a review of characteristics and applied methods. Int J Technol Assess Health Care 2018;34:537-46.

51 Jeyaraman MM, Rabbani R, Al-Yousif N, et al. Inter-Rater reliability and concurrent validity of ROBINS-I: protocol for a cross-sectional study. Syst Rev 2020;9:12.

52 Birkelbach O, Mörgeli R, Balzer F. Why and how should I assess frailty? A guide for the preoperative anesthesia clinic. Anästhesio Intensivmed Notfallmed Schmerzther 2017;52:765-76 